musculoskeletal manifestations $(\mathrm{p}=0.004)$, neuropsychiatric manifestations $(p=0.002)$, renal manifestations $(p<0.001)$ and lymphopenia $(\mathrm{p}=0.008)$ than patients with positives autoantibodies and without clinical activity.

Conclusion In our series of SLE patients with both serological manifestations and low clinical activity have lower levels of IL10 and INF1A, compared to patients with high clinical activity. This result would suggest that differences in the cytokine levels are not related to autoantibodies presence but there are other mechanisms involved in cytokine production that would also be involved in maintenance of clinical remission.

\section{PS2:31 AGE AND AN AUTOANTIBODY ALGORITHM HELPS DISTINGUISH PATIENTS WITH SYSTEMIC LUPUS ERYTHEMATOSUS FROM THOSE WITH SJOGREN'S SYNDROME}

KG Moder, CS Crowson, MR Snyder. Mayo Clinic, Department of Rheumatology, Rochester, Minnesota, USA

\subsection{6/lupus-2018-abstract.79}

Purpose Patients with Systemic Lupus Erythematosus (SLE) and Sjogren's Syndrome (SS) sometimes share common clinical features and can have similar autoantibody profiles. We evaluated a group of patients with each disease to determine which features distinguish each group.

Methods Samples $(n=1000)$ were identified based on the clinical ANA results obtained by EIA (BioRad). This testing was performed on the Triturus semi-automated platform (Grifols) in accordance with all the manufacturer's instructions. Samples were selected for this study to be distributed across the reportable range of the ANA EIA method ( $\mathrm{n}=273$ negative [<1.0 U], 225 weak positive [1.1-2.9], 250 positive [3.0-5.9], 252 strong positive [ $>5.9]$. All samples selected for this study were subsequently analysed by multiplex assay (MIA) for specific autoantibodies. The MIA testing was performed on the BioPlex 2200 (BioRad). All samples were from adults age $>18$ years).

Results Out of 1000 samples there were 227 patients with connective tissue disease including 67 with SLE and 42 with SS. We compared the SLE patients with those who had SS.

The SLE patients were younger than those with SS. (Mean age 47.4 years, median 47 , range $19-81$ vs Mean age 56.5 years, median 59, range 19-76, P 0.001). There was no difference in sex distribution ( $82 \%$ female vs $86 \%$ female).

Conclusions Somewhat surprisingly, the presence of anti-DsDna and anti-Smith antibodies was not the best way to separate the patients. The best combination of variables to distinguish SLE patients from those with SS was younger age, the presence of anti-chromatin antibodies and the absence of anti-SSA antibodies.

\section{PS2:32 CD64, FC GAMMA R I EXPRESSION LEVELS ON MONOCYTE (MCD64) AS A POTENTIAL BIOMARKER FOR NEUROPSYCHIATRIC SYSTEMIC LUPUS ERYTHEMATOSUS (NPSLE)}

${ }^{1} \mathrm{~S}$ Tsuji, ${ }^{2} \mathrm{~A}$ Kikuchi-Taura, ${ }^{1} \mathrm{E}$ Oguro, ${ }^{1} \mathrm{Y}$ OKita, ${ }^{1} \mathrm{~K}$ Kuzuya, ${ }^{1} \mathrm{H}$ Matsuoka, ${ }^{1} \mathrm{~S}$ Teshigawara, ${ }^{1} \mathrm{M}$ Yoshimura, ${ }^{1} \mathrm{Y}$ Harada, ${ }^{1} \mathrm{M}$ Matsushita, ${ }^{2} \mathrm{~S}$ Ohshima, ${ }^{2} \mathrm{Y}$ Saeki. ${ }^{1}$ Department of Rheumatology, Osaska-Minami Medical Centre, Kawachinagano, Japan; ${ }^{2}$ Department of Clinical Research, Osaska-Minami Medical Centre, Kawachinagano, Japan

10.1136/lupus-2018-abstract.80

Abstract PS2:31 Table 1

\begin{tabular}{|l|l|l|l|l|}
\hline Antibody & $\begin{array}{l}\text { SLE }(n=67) \\
\% \text { positive }\end{array}$ & $\begin{array}{l}\text { SS }(n=42) \\
\% \text { positive }\end{array}$ & P-Value & $\begin{array}{l}\text { Age adjusted } \\
\text { P-value }\end{array}$ \\
\hline chromatin & $52 \%$ & $10 \%$ & $<0.001$ & $<0.001$ \\
\hline DsDNA & $53 \%$ & $2 \%$ & $<0.001$ & 0.98 \\
\hline Ribosomal P & $13 \%$ & $2 \%$ & 0.052 & 0.27 \\
\hline RNP & $27 \%$ & $2 \%$ & 0.004 & 0.029 \\
\hline Smith & $21 \%$ & $0 \%$ & 0.002 & 0.97 \\
\hline SmRNP & $31 \%$ & $2 \%$ & $<0.001$ & 0.009 \\
\hline SSA & $49 \%$ & $93 \%$ & $<0.001$ & $<0.001$ \\
\hline SSB & $16 \%$ & $52 \%$ & $<0.001$ & $<0.001$ \\
\hline
\end{tabular}

Multivariable model to distinguish SLE from SS (reference)

\begin{tabular}{|l|l|l|}
\hline Characteristic & Odds ratio $(95 \% \mathrm{CI})$ & p-value \\
\hline Age & $0.94(0.90,0.98)$ & 0.002 \\
\hline Chr & $3.24(1.67,6.29)$ & $<0.001$ \\
\hline SS2 & $0.23(0.11,0.48)$ & $<0.001$ \\
\hline
\end{tabular}

s-statistic: 0.88 .

Interpretation: Patients with SLE are younger than patients with SS. Patients with SLE are 3 times more likely to have Chx and 5 times less likely to have SSa compared to patients with SS. 
Background and purpose Interferons (IFN), such as IFN $\alpha$ and IFN $\gamma$, are known to play a pivotal role for the pathogenesis of neuropsychiatric systemic lupus erythematosus (NPSLE). These IFNs enhance the expression of mCD64. We recently have reported a tight correlation between mCD64 expression levels and SLE disease activity index (SLEDAI) $(\mathrm{r}=0.68$, $\mathrm{p}<0.001$ ) (Lupus 24(10):1076-80, 2015) and shown that mCD64 expression is a simple and useful biomarker for evaluating disease activity in SLE patients. Although neuropsychiatric manifestations are critical to the management of SLE, there have been no precise and convenient biomarkers assessing the activity of NPSLE. In this study, we investigated the utility of mCD64 expression as a biomarker for NPSLE.

Method mCD64 expression levels were assessed quantitatively by using flow cytometry in five patients with NPSLE. The mCD64 expression levels were compared with SLEDAI and other conventional SLE activity markers, such as antidsDNA antibody, complements and cerebrospinal fluid (CSF) IL-6.

Result The NPSLE events included three headaches, two aseptic meningitis and one acute confusion. At the active phase of these NPSLE events, mCD64 expressions were significantly enhanced at the median of 38541 (range 31,693-73,287) molecules/cell compared to the treated inactive phase. Additionally, mCD64 expression was significantly higher in CSF IL-6 high (more than $4.3 \mathrm{pg} / \mathrm{ml}$ ) group than the low group $(\mathrm{p}=0.011)$. The mCD64 expression levels were significantly decreased at the inactive phase of the NPSLE after treatment $(p=0.027)$ shown in figure 1 . The changes of mCD64 expression levels correlated with SLEDAI $(r=0.74, p=0.014)$.

Conclusions mCD64 expression may be a potential biomarker for evaluating not only the disease activity but also the response of treatment in NPSLE.

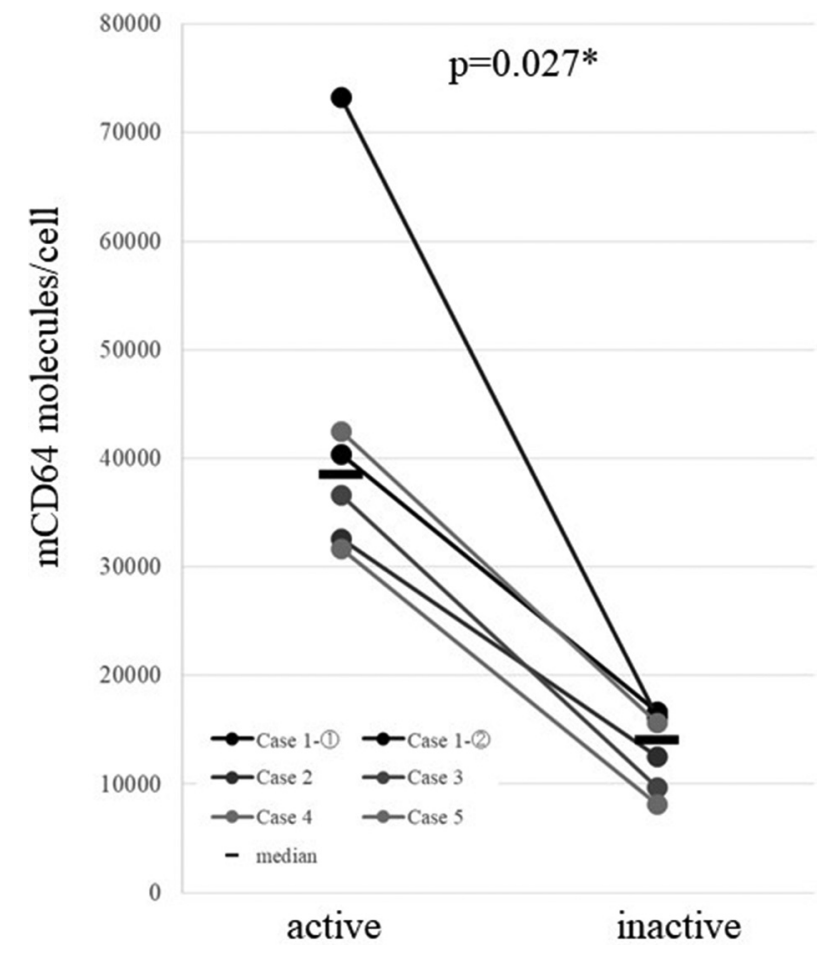

Abstract PS2:32 Figure 1 Comparision of mCD64 expression between active and inactive disease NPSLE

\section{PS2:33 MICROPARTICLES FROM SLE PATIENTS ARE A SOURCE OF INTERFERON-ALPHA}

F Miranda, C Barbati, FR Spinelli, F Ceccarelli, S Truglia, C Alessandri, G Valesini, F Conti. Dipartimento di Medicina Interna e Specialità Mediche, Reumatologia, Sapienza Università di Roma, Italy

10.1136/lupus-2018-abstract.81

Systemic lupus erythematosus (SLE) is the prototype of systemic autoimmune disorders.

Interferon alpha is a pleiotropic cytokine that can affect multiple cell types involved in lupus.

Dendritic cells (DC) have a special role in the production of IFN and are the main sources of serum interferon. IFN has the potential to dramatically influence the development, progression, and pathogenesis of SLE as it can influence the function and activation state of most major immune cell subsets and function as a bridge between innate and adaptive immunity.

Circulating microparticles (MPs) are ubiquitous in the blood of healthy individuals, These MPs play an active role in coagulation and intercellular communication and assist in activation or suppression of the immune system, depending on their parental cell origin. Changes in the concentration and/or composition of circulating Mps have been described in various autoimmune diseases, including rheumatoid arthritis (RA) systemic sclerosis (SSc) and SLE.

For SLE, the reported microparticle-related changes remain somewhat inconclusive.

To better understand the role of MPs in SLE patients, we analysed the presence of IFN-alpha on MPs surface.

MPs were isolated from citrate-treated plasma; blood cells were removed by two steps of centrifugation process $(2500 \mathrm{~g}$ for $15 \mathrm{~min}$ at $20 \mathrm{C}$ two time). The resulting platelet-poorplasma (PPP), was analysed by flow cytometry with specific antibody against IFN-alpha

20 consecutive SLE patients (10 with active lupus nephritis) and 10 sex- $^{-}$and age-matched healthy control subjects (HC) were included in the study.

We found that MPs from SLE patients carry on their surface IFN alpha.

Moreover, the percentage IFNalpha +MPs was higher in SLE patients and in lupus nephritis patients than in HC, but there was not significant difference between patients with and without renal involvement.

The results of the present study show for the first time the presence of IFN-alpha on MPs surface.

We may assume that INF+MPs derive from DC. In lupus nephritis patients the increased recruitment of DC was at tubular interstitial level, with subsequent IFN-alpha production. Interestingly, MPs (containing RNA and DNA) could stimulate type I IFN production in plasmacytoid dendritic cells and subsequently the release of MPs from DC.

\section{PS2:34 THE EFFECT OF AUTO-ANTIBODY POSITIVITY ON DAMAGE AND DEATH IN A LARGE COHORT OF PATIENTS WITH SYSTEMIC LUPUS ERYTHEMATOSUS: A 40-YEAR FOLLOW-UP STUDY}

BS Bernstein, C Wincup, T Mcdonnell, B Tejera Segura, A Rahman. Centre for Rheumatology, UCL, London, UK 